\title{
Neuartiges Antriebskonzept zum Fahren, Lenken und Heben
}

\author{
Dipl.-Ing. Manuel Weber, Dipl.-Ing. Christian Vorwerk \\ Universität Stuttgart, \\ Institut für Fördertechnik und Logistik \\ Maschinenentwicklung und Materialflussautomatisierung
}

\begin{abstract}
Im Lagerbereich von intralogistischen Systemen werden zur Handhabung von Waren unterschiedliche technische Komponenten eingesetzt, wie z.B. Horizontalkommissionierer, elektrische Gabelhubwagen oder Fahrerlose Transportsysteme. Es gibt jedoch noch zahlreiche Aufgaben bei denen eine neue kompaktere und flexiblere Transporttechnik die Arbeit deutlich erleichtern würde, so z.B. beim Rangieren auf LKW-Ladebordwänden und in beengten Lager- oder Kaufhausbereichen. Es wurde eine neuartige Transportvorrichtung entwickelt, bei der sowohl die Fahr-, Lenk- und Hubfunktionen als auch die benötigte Energieversorgungs- und Steuerungstechnik implementiert sind. Die Transportvorrichtung besitzt dabei ungefähr die Abmessungen einer Gabel eines Gabelhubwagens.
\end{abstract}

\section{$1 \quad$ Einleitung}

Zum Transport von Waren sind u.a. Fahrerlose Transportfahrzeuge (FTF) und elektrische (manuell zu bedienende) Gabelhubwagen Stand der Technik. FTFs lassen sich prinzipiell unterscheiden in Fahrzeuge, die eine eigene aktive Lastaufnahmevorrichtung besitzen und Fahrzeuge die über eine externe Handhabungseinrichtung be- und entladen werden müssen. Die Integration einer Lastaufnahmevorrichtung steigert häufig die Komplexität der Fahrzeuge und erhöht den benötigten Bauraum, da zusätzliche Antriebe und eine Hubvorrichtung angebracht werden müssen. Aufgrund zahlreicher Aufgaben, wie z.B. beim Rangieren auf LKW-Ladebordwänden oder in beengten Lager- oder Kaufhausbereichen, die mit aktueller Technik nicht zufriedenstellend bewerkstelligt werden, ergibt sich die Forderung nach einer kompakten Antriebstechnik, bei der alle Antriebskomponenten innerhalb einer Gabel eines Gabelhubwagen oder ähnlichem Platz finden. Alle Fahrzeuge die ferngesteuert oder gar autonom betrieben werden benötigen ein auf ihr Einsatzgebiet angepasstes Lenkkonzept.

Am Institut für Fördertechnik und Logistik wurde ein neuartiges Hubkonzept entwickelt, welches leistungsfähig genug ist, eine beladene Europalette anzuheben und dennoch so kompakt ausgeführt ist, um im Bauraum einer Hubgabel Platz zu finden. 


\section{$2 \quad$ Entwicklung einer neuartigen Transportvorrichtung}

Die VDI-Richtlinie 2510 bietet zum Stand der Technik einen Überblick unterschiedlicher Fahrwerkvarianten und deren möglichen Fahrbewegungen, siehe Abbildung 1.

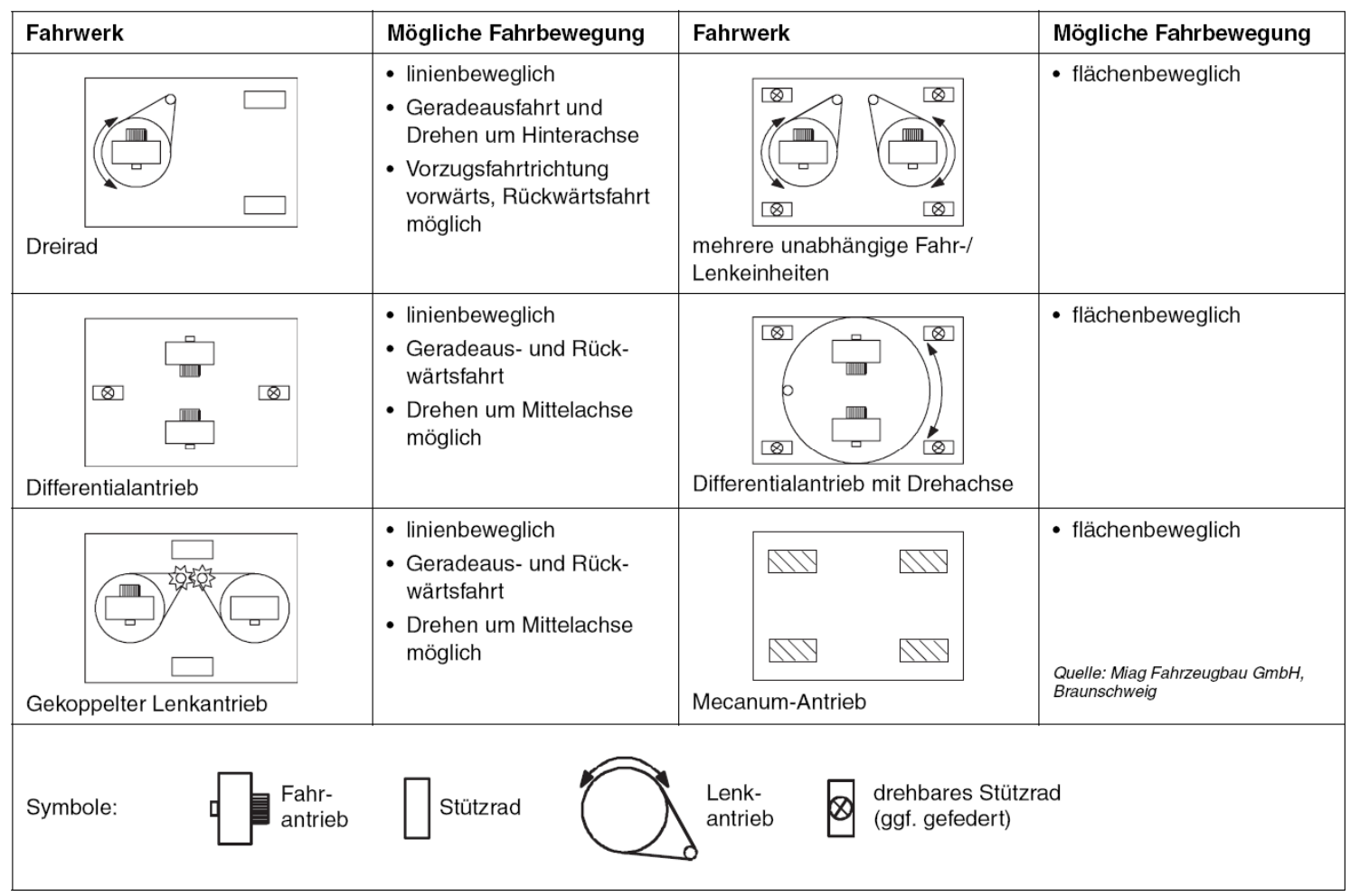

Abbildung 1: Mögliche Fahrbewegungen unterschiedlicher Fahrwerke [VDI05]

Aktuell sind durch den Einsatz moderner Lenkprinzipien bei FTF flächenbewegliche Fahrbewegungen möglich. Teilweise kommen die Fahrzeuge bereits ohne einen eigenen Lenkantrieb aus, z.B. omnidirektionale Fahrzeuge (Mecanum Antrieb [VDI05] oder Fahrzeuge mit Omniwheels). Aufgrund der Vielzahl an unterschiedlichen Lenkprinzipien lässt sich (z.T. auch durch Kombination mehrerer Verfahren) für nahezu jede Anwendung eine passende Lenkung finden.

Zur Realisierung des Hubvorgangs muss eine Wandlung von zumeist elektrischer Energie in translatorische und damit in potentielle Energie erfolgen. Hierfür gibt es u.a. folgende Möglichkeiten:

- Linearmotor,

- E-Motor mit Hub-Spindelgetriebe,

- E-Motor mit Hub-Spindelgetriebe und Scherenkinematik,

- Elektrohydraulisch oder

- Elektropneumatisch. 


\subsection{Neuartiges Antriebskonzept zum Fahren, Lenken und Heben}

Zum Lösen oben genannter Aufgabenstellung wurde ein neuartiges Antriebskonzept entwickelt, das die Funktionen Fahren, Lenken und Heben in einer extrem kompakten Bauweise erfüllen kann. Durch Kombination der Lenkfunktion mittels eines Differentialantriebs mit Drehachse und der Hubfunktion als Hubspindel entsteht ein elektromotorischer Antrieb, der erstmalig diese Funktionen gleichzeitig erfüllen kann, siehe Abbildung 2. Bei der Drehschemellenkung mit Differentialantrieb ergibt sich bei mehrfacher Anordnung (mindestens drei Elemente, die nicht auf einer Linie liegen) ein flächenbewegliches Fahrzeug. Bei dem neu entwickelten Antriebssystem gelingt es, die Hub-, Fahr- und Lenkbewegung zu kombinieren, um sowohl dem Fahrzeug eine bestimmte Fahrtrichtung vorzugeben und es anzutreiben als auch die Hubplattform in die Höhe zu bewegen.

Mittels der Transportvorrichtungen, insbesondere bei einer Verwendung als aus zwei Elementen zusammen wirkenden Hebe- und Transportvorrichtung für Paletten, ist eine völlig geänderte Art des Manövrierens gegenüber handelsüblichen Gabelhubwagen möglich. So kann beispielsweise seitlich in eine Palette eingefahren und dann die Palette nach dem Anheben senkrecht zur Einfahrrichtung transportiert werden. Eine Palette kann auch um ihre Hochachse auf der Stelle gedreht werden.

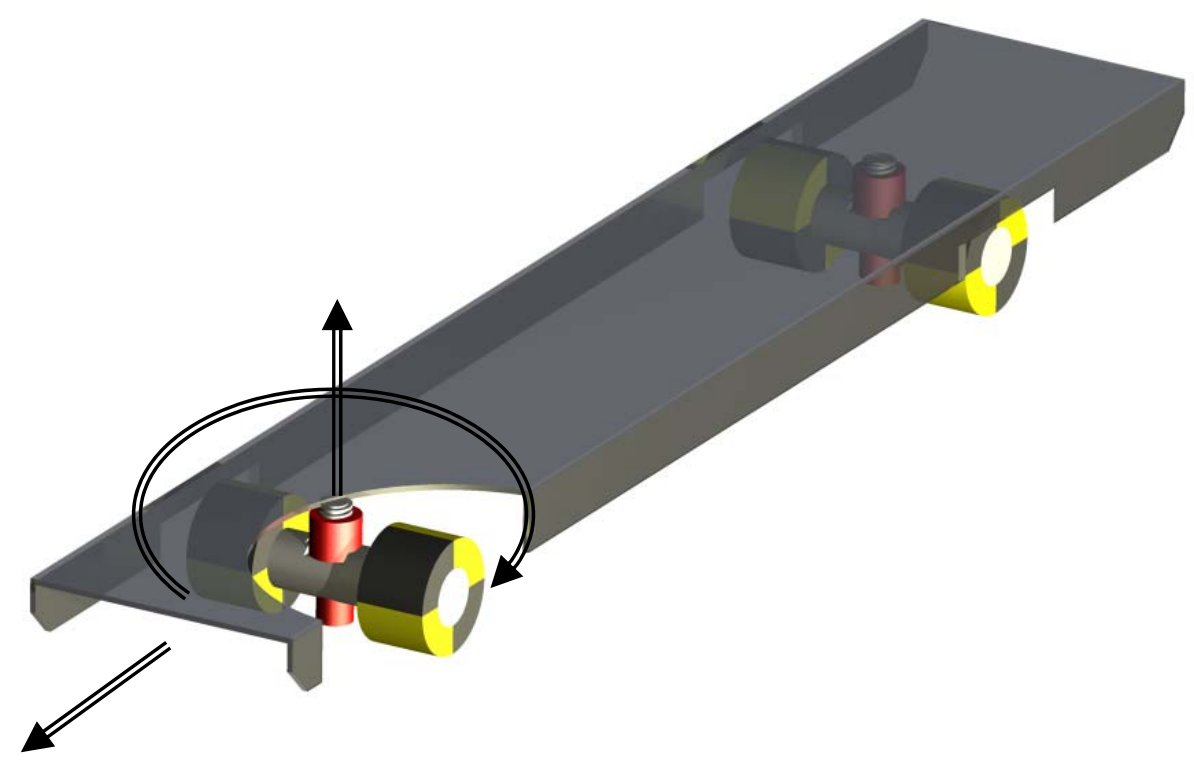

Abbildung 2: Bewegungsmöglichkeiten einer Transportvorrichtung

Die entwickelte Transportvorrichtung, insbesondere die Antriebseinheiten zum Fahren, Lenken und Heben werden ferngesteuert betrieben. Weiterhin werden zur Steuerung Signale benötigt, die die Position der Transportvorrichtung zur Orientierung im Raum, in Relation zu Hindernissen, Fahrzielen oder anderen Transportvorrichtungen angibt. 


\subsection{Beschreibung des Wirkprinzips}

Bei der Transportvorrichtung sind Antrieb, Steuergerät und Stromversorgung innerhalb eines Raumbereichs des Trägerteils angeordnet, so dass das Trägerteil den Ladungsträger vollständig unterfahren kann. Eine mechanische Verbindung zwischen zwei Transportvorrichtungen ist nicht vorgesehen. Das Trägerteil unterschreitet die lichten Abmessungen des Ladehilfsmittels oder eines Ladungsträgers bspw. einer Euro-Palette. Auf diese Weise können zwei Transportvorrichtungen wie die Lastgabel eines Hubwagens eingesetzt werden und dort auch zu Lagerzwecken (z.B. auf einer LKW-Ladefläche) ohne weiteren Raumbedarf untergebracht werden. Zum Transport von Paletten wird die Transportvorrichtung paarweise eingesetzt, wobei eine entsprechende Steuerung der beiden Transportvorrichtungen und deren Fernsteuerung koordiniert erfolgen kann, indem die beiden Transportvorrichtungen (zumindest beim Transport des Ladungsträgers) parallel und in einem festen Abstand voneinander als ein Paar gesteuert werden, während beim Rangieren ohne Ladung jede Transportvorrichtung für sich gesteuert werden kann.

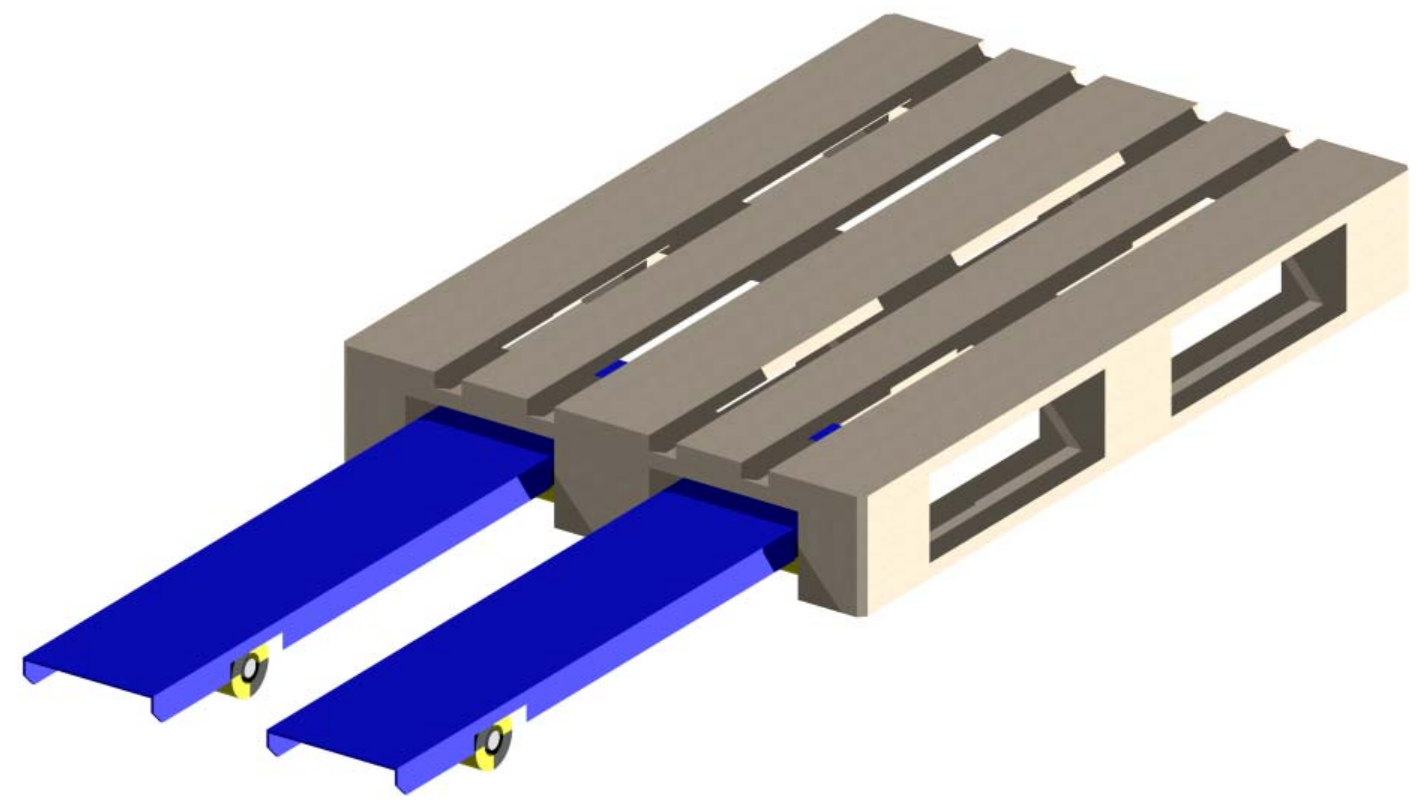

Abbildung 3: Transportvorrichtung beim Unterfahren einer Palette

Abbildung 3 zeigt in schematischer Darstellung zwei identische Ausführungsformen zweier zueinander parallel im Abstand von Aufnahmeöffnungen einer Palette angeordneter Transportvorrichtungen, die als eine Einheit zur Aufnahme des Ladungsträgers zusammenwirken. 


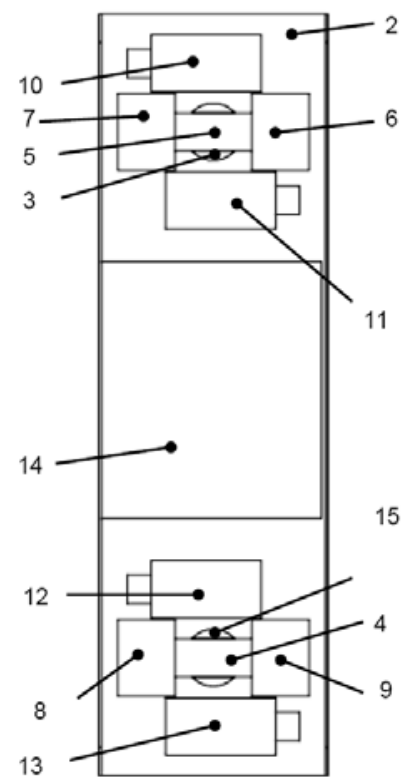

Abbildung 4: Sicht auf Transportvorrichtung von unten [DE07]

Die Transportvorrichtungen kommunizieren drahtlos miteinander bzw. einzeln mit der Leitstelle. Werden zwei Transportvorrichtungen als ein Paar eingesetzt übernimmt eine (der Transportvorrichtungen) die Master-Funktion und kommuniziert mit der Leitstelle, die zentral die Steuerung einer oder mehrerer einzelner beziehungsweise zu Paaren zusammengefasster Einheiten von Transportvorrichtungen übernimmt.

Jede einzelne Transportvorrichtung weist ein Trägerteil 2 auf, das einen U-förmigen Querschnitt besitzt, wodurch die erforderliche Stabilität gewährleistet ist, siehe Abbildung 4. Die Länge ist je nach Abmessungen der Ladehilfsmittel oder Ladungsträger wählbar, bzw. bei unterschiedlichen Anwendungen auch teleskopierbar ausführbar. Die Trägerteile nehmen die übrigen Bauteile an ihrer Unterseite auf. Die beiden Achsen 4, 5 sind mit Getrieben 3 bzw. 15, die eine Drehbewegung in einen Hub wandeln (beispielsweise eine Spindelmutter mit Spindel) mit den Trägerteilen verbunden. An den Achsen befinden sich die Räder 6, 7, 8, 9. Die Getriebe können einen Freiwinkel aufweisen, bei denen während einer Verdrehung kein Hub resultiert. Ferner ist es denkbar eine Kupplung zwischen Achse und Spindelmutter zu montieren, um gezielt eine Übertragung des Drehmomentes steuern zu können. Dadurch wären dann Drehbewegungen (auch über $90^{\circ}$ ) ohne eine Hubbewegung möglich. Jedes einzelne Rad wird von einer Antriebseinheit 10, 11, 12, 13 (beispielsweise einem Elektromotor mit Getriebe) angetrieben. Die Antriebseinheiten sind in dem gezeigten Ausführungsbeispiel jeweils vor und hinter den Achsen angeordnet. Die Antriebseinheiten sind mit den Achsen fest verbunden und werden bei einer Verdrehung dieser mit gedreht. Die - nicht dargestellte Verbindung zwischen den Antriebseinheiten und den Rädern erfolgt über einen Riementrieb. Die Antriebseinheiten werden von einer zwischen den Achsen vorgesehenen Steuer- und Versorgungseinheit 14 angesteuert und mit elektrischer 
Energie versorgt. Zur Stromübertragung wird ein Drehübertrager eingesetzt. Die Steuer- und Versorgungseinrichtung besteht aus

- einer Kommunikationseinheit, die drahtlos Befehle sendet und empfängt,

- einem internen Rechner mit einer implementierten Software die Steuersignale der Antriebseinheiten umsetzt und

- einer Stromversorgungseinheit, die aus Akkumulatoren besteht und die elektrische Energie für die Antriebseinheiten und die Steuereinheit zur Verfügung stellt.

Sämtliche Steuer- und Versorgungsleitungen zwischen den einzelnen elektrischen Komponenten sind nicht dargestellt.

\subsubsection{Fahren}

Zur Geradeausfahrt der Transportvorrichtung werden alle Antriebseinheiten in gleichem Drehsinn (bezogen auf die Achsen) bestromt und damit alle Räder in die entsprechende Fahrtrichtung gleichsinnig angetrieben.

\subsubsection{Lenken}

Bei der beschriebenen Lösung entfällt ein separates Lenkgetriebe. Vielmehr erfolgt eine Lenkung der vorgeschlagenen Transportvorrichtung durch Verdrehen der Achse durch unterschiedliche Antriebsgeschwindigkeiten beziehungsweise Drehrichtungen der Räder (Differentialantrieb). Zum Lenken wird je nach gewünschtem Lenkprinzip beispielsweise das kurvenäußere Rad 6 mit einer höheren Drehzahl angetrieben als das kurveninnere Rad 7. Um die Kurvenfahrt einzuleiten wird zunächst die Achse 5 verdreht. Die Räder der zweiten Achse 4 können sich ähnlich einer PKWHinterachse verhalten und nur den Drehzahlunterschied ausgleichen - ähnlich einem Hinterachsdifferential. Alternativ werden die Räder zunächst derart angetrieben, dass sich ein betragsmäßig gleich großer Winkel wie an Achse 5 einstellt, dieser jedoch in die andere Richtung zeigt. Ist der oben beschriebene Lenkwinkel an Achse 5 erreicht, so werden die beiden Räder 8 und 9 genauso angetrieben wie die Räder 7 und 6. Die Räder 7 und 8 sowie die Räder 6 und 9 bewegen sich nun jeweils auf identischen Bahnkurven. Durch die vorhandene Allradlenkung ergeben sich unterschiedliche Lenkmöglichkeiten, so z.B. auch der Hundegang, siehe Abbildung 5. Dies ermöglicht ein Verfahren seitlich zur eigentlichen Fahrzeuglängsrichtung. Ebenso lässt sich der Momentanpol mittig in Fahrzeuglängsrichtung verschieben, wodurch sich ein geringer Wenderadius ergibt. Weitere Bewegungsmöglichkeiten sind in Abbildung 5 dargestellt. Je nach Fahrtrichtung lässt sich bei der Transportvorrichtung die Vorder- oder Hinterachse lenken. Somit lässt sie sich entweder wie ein PKW oder wie ein Gabelstapler manövrieren. 

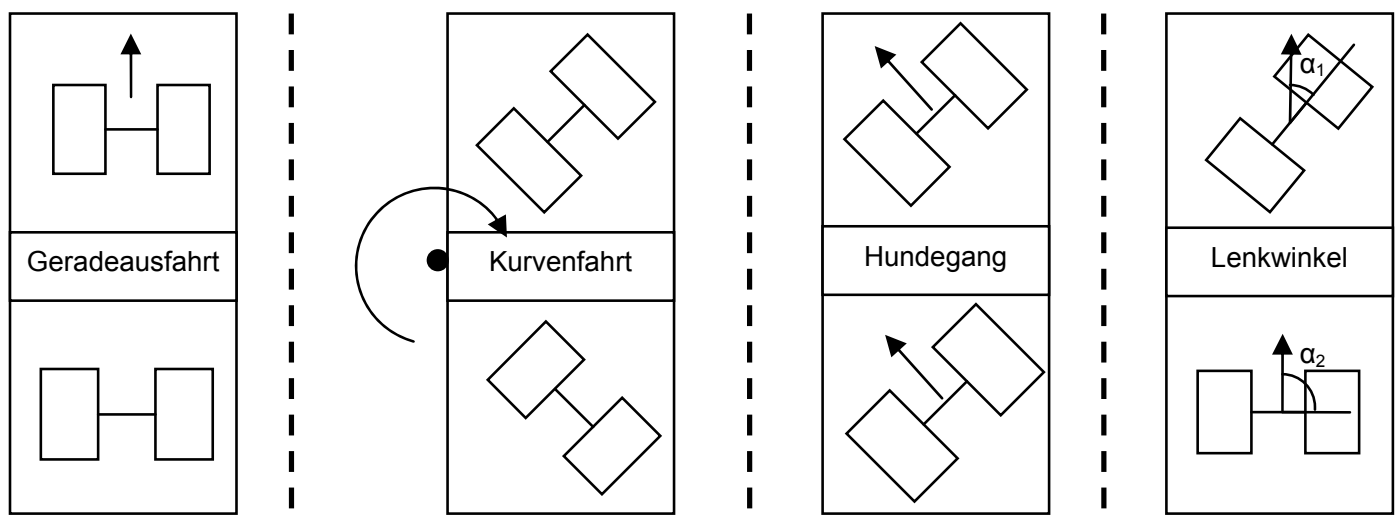

Abbildung 5: Fahrzustand einer Transportvorrichtung

Der Lenkeinschlag führt jedoch bei einem Drehschemel immer zu einer Verringerung der „Standfläche“ [Mat07]. Dadurch sinkt bei einzelnen Transportvorrichtungen die Stabilität. Um ein Kippen einer Transportvorrichtung (wenn $\alpha_{1}$ und $\alpha_{2}$ sehr klein) zu verhindern muss sichergestellt werden, dass z.B. bei einem Lenkwinkel an Achse 4 mit $\alpha_{1}<45^{\circ}$ der Lenkwinkel $\alpha_{2}$ an Achse 5 mindestens $45^{\circ}$ beträgt. Werden zwei der Gabeln verwendet und bspw. über eine Palette "gekoppelt", so ergibt sich eine flächenbewegliche Transportvorrichtung, mit beliebig wählbarem Drehpunkt, z.B. Drehen um den Mittelpunkt einer Palette, siehe Abbildung 6.
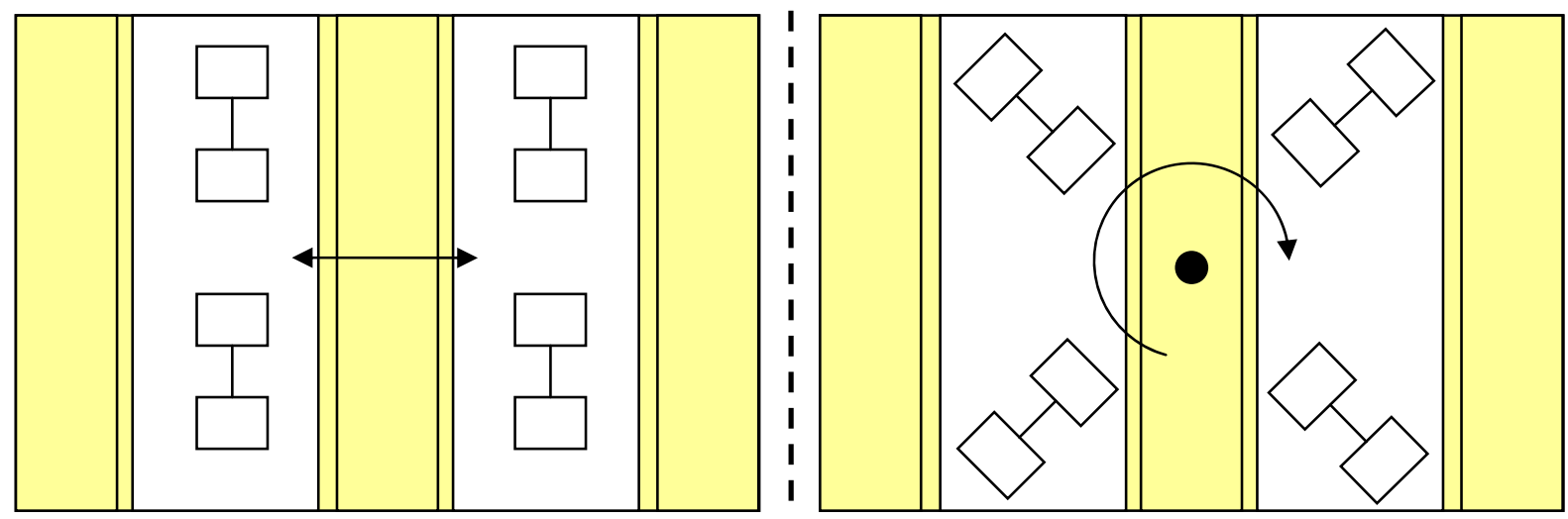

Abbildung 6: Fahrzustand zweier Transportvorrichtungen gekoppelt über Palette

\subsubsection{Heben}

Zwischen den Achsen und dem Trägerteil sind Hubspindeln angebracht. Durch gegenläufiges Verdrehen der an einer Achse angeordneten Räder werden die Achsen und somit auch die Spindelmutter verdreht, wodurch sich je nach Drehrichtung der Antriebseinheiten ein Anheben oder ein Absenken des Trägerteils ergibt. Beim Heben einer einzelnen Transportvorrichtung muss auf eine phasenverschobene Drehung der beiden Achsen einer Plattform geachtet werden. Die Drehwinkel der Achsen $\alpha_{1}$ und $\alpha_{2}$ sollten beim Heben jeweils eine Differenz von ca. $90^{\circ}$ aufweisen, vgl. Abbildung 5 . 


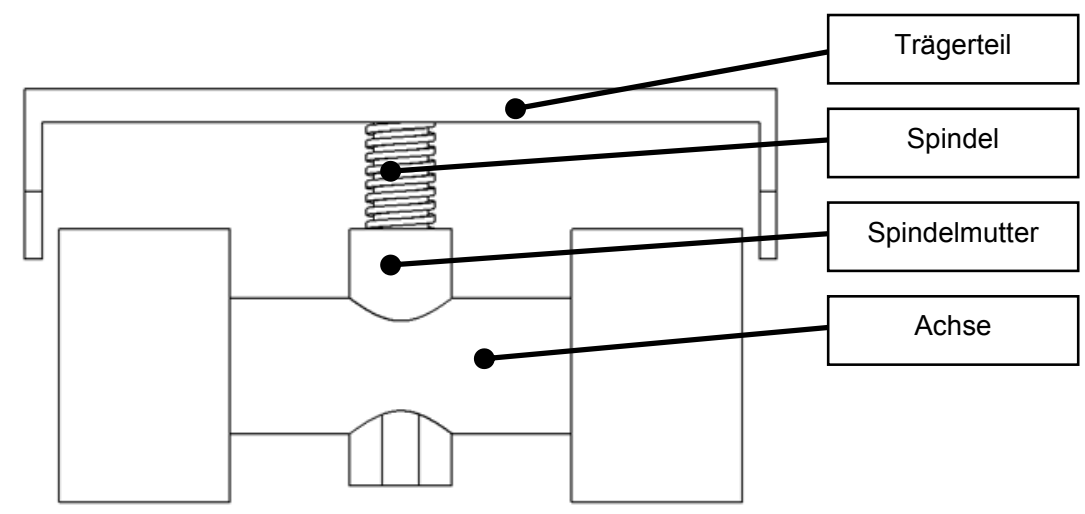

Abbildung 7: Transportvorrichtung mit angehobenem Trägerteil

Abbildung 7 zeigt die Transportvorrichtung mit angehobenem Trägerteil nach mehreren Umdrehungen der Achse um ihre Hochachse durch gegenläufigen Antrieb der Räder. Die Spindelmutter wird dadurch verdreht und hebt das Trägerteil und eine gegebenenfalls darauf befindliche Last an.

\subsection{Leistungsbetrachtung}

Zur Betrachtung inwieweit sich die Motoren sowohl als Antriebs-, Lenk- als auch als Hubantrieb eignen wurden die erforderlichen Leistungsparameter bestimmt und miteinander verglichen. Bei einer Nutzlast von $400 \mathrm{~kg}$ je Transportvorrichtung ergeben sich Leistungsdaten für die Antriebseinheiten wie in Abbildung 8 dargestellt.

\begin{tabular}{|l|l|l|l|}
\hline & $\begin{array}{l}\text { Geschwindig- } \\
\text { keit }[\mathrm{m} / \mathrm{s}]\end{array}$ & $\begin{array}{l}\text { Drehzahl } \\
{[1 / \mathrm{min}]}\end{array}$ & Leistung [W] \\
\hline Fahren & 1,0 & 235 & 155 \\
\hline Heben & 0,01 & 300 & 123 \\
\hline
\end{tabular}

Abbildung 8: Technische Daten einer Transportvorrichtung

Aus Abbildung 8 wird ersichtlich, dass ein sinnvoller Einsatzbereich für die Motoren sowohl zum Heben als auch zum Fahren vorliegt.

\subsection{Vor- und Nachteile des neuartigen Konzepts}

Die größten Vorteile der neuartigen Transportvorrichtung sind die flexible Bewegungsmöglichkeit, sowie die kompakte Bauweise, wodurch das Unter- und Durchfahren von Paletten möglich wird. Weiterhin werden für den Bau und die Realisierung der Funktionen Fahren, Lenken und Heben identische Antriebskomponenten verwendet. Diese Antriebskomponenten (Motoren, Getriebe) besitzen einen hohen Auslastungsgrad und werden alle gleichmäßig belastet. Dadurch können bei Wartungs- und Serviceintervallen die anstehenden Maßnahmen für alle Motoren gleichermaßen angewendet werden. Ein weiterer Vorteil besteht darin, dass die Transportvorrichtung ohne eine separate (reine) Hubeinheit, sowie ohne ein Lenkgetriebe auskommt. Die Transportvorrichtungen lassen sich beispielsweise im manuellen Betrieb einsetzen, quasi als hochflexibler voll (c) 2010 Logistics Journal : Proceedings - ISSN 2192-9084 
elektrischer Gabelhubwagen, wodurch sich auch in engen Gassen (z.B. in Kaufhäusern beim Nachschub) eine höhere Manövrierfähigkeit ergibt als bei Standard gelenkten „Dreirad“-Hubwagen. Weiterhin ist ein automatischer Betrieb denkbar, wobei durch eine geeignete Sicherheitsüberwachung und Navigation mehrere Hubwagen im Pulk fahren können. Einen weiteren Vorteil stellen die vier direkt angetriebenen Räder pro Transportvorrichtung dar. So kann selbst bei schwierigen Reibverhältnissen eine Kraftübertragung (auch beim Heben) sichergestellt werden. Ferner herrscht durch die angepasste Drehzahl zwischen innerem und kurven äußerem Rad bei Kurvenfahrt nur ein geringer Schlupf. Bei Geradeausfahrt reduziert sich der Schlupf auf einen üblichen Bereich. Bei einer dem aktuellen Betriebszustand (eine Transportvorrichtung allein oder zwei Transportvorrichtungen gekoppelt) angepassten Steuerung verhält sich das Fahrzeug völlig stabil und ein Kippen kann ausgeschlossen werden.

Aufgrund der oben beschriebenen Verringerung der Standfläche beim Lenkeinschlag ist ein omnidirektionales Fahren nur mit Palette o.ä. zum Verbinden zweier Transportvorrichtungen möglich. Ein weiterer Nachteil besteht in dem erforderlichen Drehübertrager zur Übertragung der Antriebsenergie und Steuersignale. Die Steuerung der Transportvorrichtung wird aufwendiger als bei klassischen FTF mit einem Lenkgetriebe. Da die Spindel das einzige Verbindungselement zwischen Achse und Trägerteil darstellt, muss sie sämtliche Querkräfte übertragen. So können z.B. hohe Schwellen schlechter überfahren werden, als mit einem klassischen Gabelhubwagen. Deshalb sollte das Einsatzgebiet in erster Linie im Indoor-Bereich liegen.

\section{Anwendungen in der Intralogistik}

Die Transportvorrichtungen sind überall dort einsetzbar, wo kompakte voll manövrierfähige Hubvorrichtungen erforderlich sind oder sich Fahrzeuge in einem abgesperrten Sicherheitsbereich automatisiert bewegen.

\subsection{Pulk-Enladung von LKW}

Beim Entladen von LKWs werden beispielsweise handbedienbare Elektrohubwagen oder Elektro-Stand-Gabelhochhubwagen eingesetzt. Mit den vorgestellten Transportvorrichtungen ist es denkbar, zwei Reihen Paletten einer LKW Ladung auf einmal zu entladen oder zu beladen, siehe Abbildung 9. Jedoch muss auch hier die Sicherheit von Personen im Fahrbereich sichergestellt werden [EN97]. Es ist beispielweise denkbar, dass ein Mitarbeiter mehrere dieser Transportvorrichtungen steuert. Somit könnten die Mitarbeiter von den durch stark beschleunigende ElektroStand-Gabelhochhubwagen entstehenden Belastungen entlastet werden. Auch wenn mit den neu entwickelten Transportvorrichtungen keine so hohen Geschwindigkeiten und Beschleunigungen zu erreichen sind, lassen sich dennoch durch die parallele Verwendung ebenfalls hohe Durchsatzzahlen erzielen. 


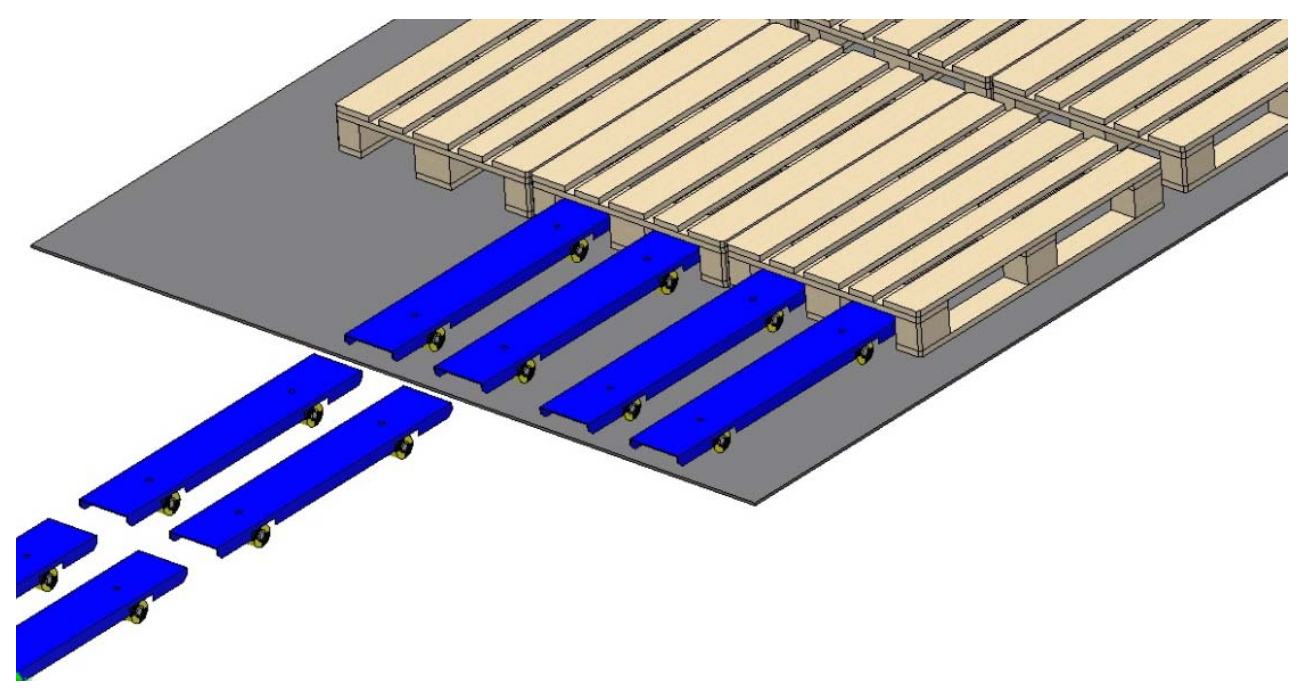

Abbildung 9: Pulkweiser Transport von Paletten

\subsection{Mitnahme von Transportgeräten auf LKWs}

Vereinfachen würde sich mit der vorliegenden Entwicklung auch die Mitnahme von Transportgeräten auf einem LKW. Die beschriebenen Vorrichtungen lassen sich beim Transport von z.B. Europaletten immer im Lichtraumprofil der Palette mittransportieren, so dass kein zusätzlicher Platz auf der Ladefläche benötigt wird.

\subsection{Blocklager}

Durch den Einsatz mehrerer Transportvorrichtungen lässt sich in einem Blocklager durch mehrere (gleichzeitig ablaufende) Umschichtvorgänge eine bessere Zugänglichkeit erreichen. Es wäre nur eine freie Gasse erforderlich und durch seitliches Verschieben wird der Zugang auch zu den hinteren Paletten ermöglicht, siehe Abbildung 10.

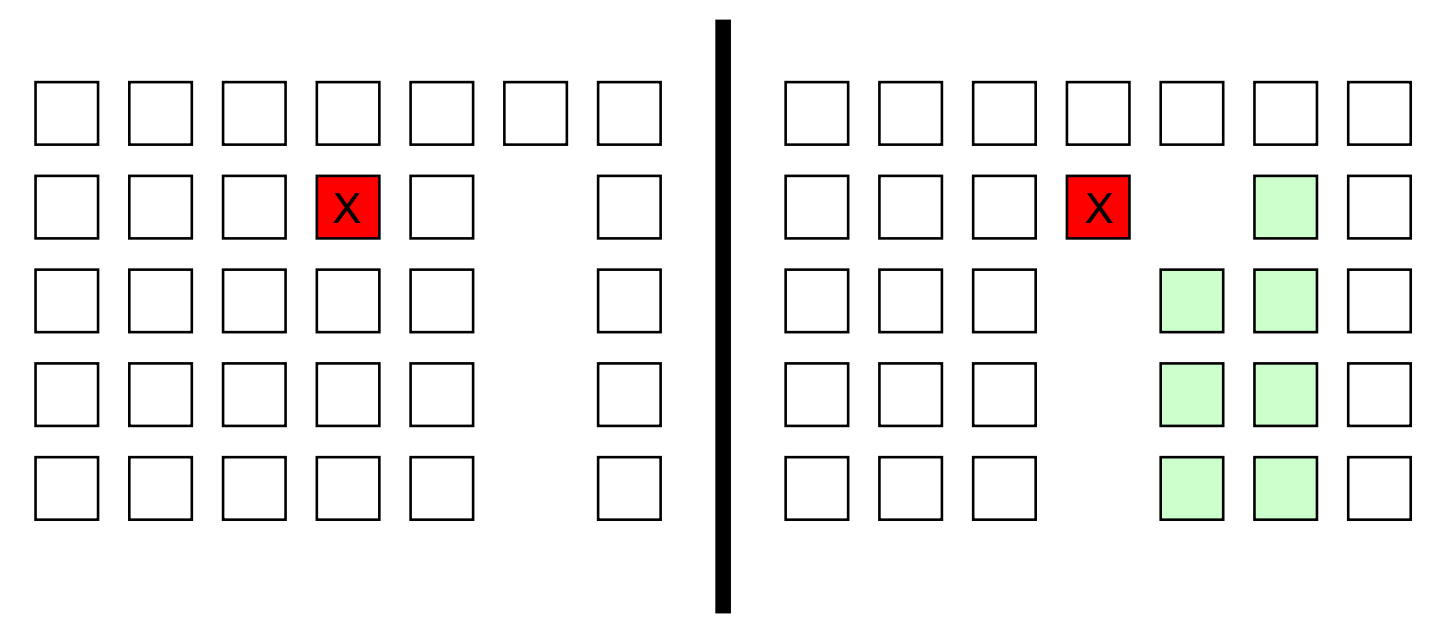

Abbildung 10: Umschichtungen in einem Blocklager 


\subsection{Virtuelle gekoppelte Routenzüge}

Bei bisherigen Routenzügen für Großladungsträger (GLT) besteht beim Aus- bzw. Einkoppeln von Anhängern entweder ein konstruktiv erhöhter Aufwand oder es erfordert im Einsatz von dem Bediener einen erhöhten manuellen Aufwand. Diese Problemstellung könnte durch eine virtuelle Kopplung von mehreren Paaren von Transportvorrichtungen in einfacher Weise gelöst werden, siehe Abbildung 11. Ein weiterer Vorteil besteht darin, dass die GLTs nicht extra auf einen Anhänger geladen werden müssen, sondern direkt aus einer Bodenlagerung entnommen werden können.

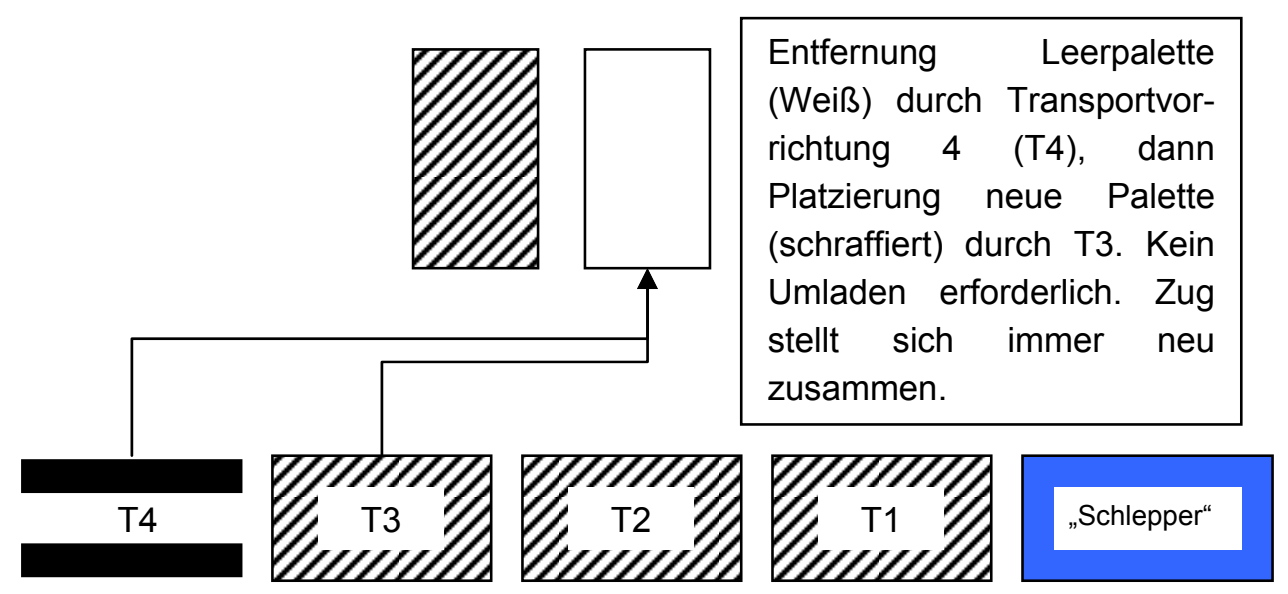

Abbildung 11: Virtuell gekoppelte Routenzüge

\section{$4 \quad$ Zusammenfassung und Ausblick}

Es wurde eine neuartige Transportvorrichtung entwickelt, die in der Intralogistik durch ihre Flexibiliät (einzeln einsetzbar, paarweise gesteuert, automatisch gesteuert, automatisch im Pulk gesteuert), ihrer Kompaktheit und Manövrierbarkeit neue Möglichkeiten bietet. Insbesondere in engen Bereichen oder dort wo es auf eine funktionale Flexibilität (siehe Beispiele Pulk, Blocklager, Routenzug) ankommt, kann diese Entwicklung für eine Erleichterung im innerbetrieblichen Materialfluss sorgen. Die Fahrzeuge bilden eine Kombination aus FTF und elektrischen Gabelhubwagen und stellen eine neue Fahrzeugkategorie dar.

Die nächsten Entwicklungsschritte sind neben der Ausarbeitung der mechanischen Konstruktion, die Auslegung der Energieversorgung und die Auswahl der Antriebskomponenten. Einen weiteren Hauptpunkt stellen die Steuerung, insbesondere die Programmierung der einzelnen Bewegungszustände sowie die Kopplung von mehreren Fahrzeugen dar. Neben der Steuerung muss auch noch ein Konzept für die Ortung und Navigation ausgewählt bzw. entwickelt werden, damit die Fahrzeuge sich ohne manuellen Eingriff durch die jeweiligen Lagerbereiche steuern lassen. 


\section{$5 \quad$ Literatur}

[DE07] DE102007046868 A1: Transportvorrichtung für Ladungsträger und Verfahren zu deren Steuerung

[EN97] DIN EN 1525 Sicherheit von Flurförderzeugen - Fahrerlose Flurförderzeuge und ihre Systeme. Berlin: Beuth Verlag, 1997.

[Mat07] Matschinsky, Wolfgang: Radführungen der Straßenfahrzeuge. Berlin: Springer-Verlag, 2007.

[VDI05] VDI 2510 Fahrerlose Transportsysteme. Berlin: Beuth Verlag, 2005. 